\title{
Feelings of familiarity and false memory for specific associations resulting from mugshot exposure
}

\author{
Alan W. Kersten ${ }^{1}$ • Julie L. Earles ${ }^{1}$
}

Published online: 29 July 2016

(C) Psychonomic Society, Inc. 2016

\begin{abstract}
This research reveals that mugshot viewing accompanied by questions about an action can cause young adults to associate the pictured person and the queried action, leading to later false recollection of having seen that person perform that action. In contrast, mugshot viewing in older adults can lead to vague feelings of familiarity for the pictured person, encouraging older adults to later falsely recognize the pictured person performing any familiar action. Participants viewed events involving actors performing different actions and then were asked verbal questions about which actor had performed each action, with each question accompanied by mugshots of potential "perpetrators" of the action. In a later recognition test, older adults were more likely to falsely recognize a novel conjunction of a familiar actor and action if they had seen a mugshot of that actor, regardless of whether the mugshot had accompanied a question about that action. In contrast, young adults were more likely to falsely recognize a conjunction event only if it involved an actor whose mugshot had accompanied a question about that particular action. This effect remained when the analysis was limited to trials involving actors whose mugshots had not been previously selected, implicating false recollection rather than commitment effects.
\end{abstract}

Alan W. Kersten

akersten@fau.edu

1 Department of Psychology, Florida Atlantic University, Boca Raton, FL 33431-0991, USA
Keywords False memory · Recollection · Familiarity · Recognition memory · Aging · Eyewitness testimony

On October 27, 1985, a woman was raped in her home in Alexandria, Virginia (Scheck, Neufeld, \& Dwyer, 2000). She told police that the room was too dark for her to clearly see the perpetrator's face, but two neighbors reported seeing a man named Walter Snyder outside of his home, just across the street from the victim's house, shortly before the attack. The police questioned Snyder and took his photograph, which was later included in a set of mugshots that were shown to the victim. The victim did not identify Snyder as the perpetrator, but she lingered on Snyder's photo and noted that his eyebrows looked familiar. Months later, when Snyder returned to the police station, the investigator asked the victim to come in to view a potential suspect. When she saw Snyder in the lobby, she expressed a great deal of apprehension to the receptionist, who quickly ushered her downstairs to meet the detective. The victim told the detective, "The man who raped me is upstairs in the lobby" (p. 51).

Snyder was convicted and sentenced to 45 years in prison, largely on the basis of the victim's testimony. Years later, Snyder's family won approval to submit semen samples from the crime scene to recently developed DNA testing techniques. Three separate tests were conducted, all of which excluded Snyder as the rapist. Snyder was eventually pardoned by the governor after having served nearly seven years in prison.

This case demonstrates the hazards associated with presenting mugshots of suspects to eyewitnesses or the victims of a crime. Although mugshot viewing can be a useful investigative tool when the perpetrator is present among the mugshots (Lindsay, Nosworthy, Martin, \& Martynuck, 1994), it may also have the effect of contaminating eyewitness memory for 
the criminal event. Attending to a mugshot of an innocent person, either because the pictured individual resembles the actual perpetrator or because the pictured individual is familiar for other reasons, may cause the eyewitness to later remember having seen the pictured individual commit the crime.

The mugshot of Walter Snyder likely appeared familiar to the victim because she had seen him in passing in the neighborhood. Although she claimed not to have had much interaction with the neighbors, she reported seeing him across the street washing his car two weeks after the attack, and thus it is likely that she had also seen him on other occasions. Snyder's case thus seems to represent an example of unconscious transference, in which a person encountered in an innocent context becomes associated with the actions of a perpetrator of a crime (Loftus, 1976). Unconscious transference in this case appears to have been facilitated by the presentation of a mugshot of the innocent person along with questions about the criminal actions of the perpetrator, perhaps linking the innocent person with the crime.

Several mechanisms have been proposed to explain the influences of mugshot viewing on subsequent false identification. One is the commitment effect (Gorenstein \& Ellsworth, 1980), occurring when a witness selects a face from a set of mugshots. When that same face appears in a later lineup, this earlier commitment causes the witness to be more likely to select that face again. Although the commitment effect has been documented in numerous studies (e.g., Blunt \& McAllister, 2009; Brigham \& Cairns, 1988; Dysart, Lindsay, Hammond, \& Dupuis, 2001; Goodsell, Gronlund, \& Neuschatz, 2015; Goodsell, Neuschatz, \& Gronlund, 2009; Haw, Dickinson, \& Meissner, 2007; Valentine, Davis, Memon, \& Roberts, 2012), it cannot explain why mugshot viewing would cause an eyewitness to remember having seen a pictured individual commit a crime when the eyewitness did not in fact select the mugshot of that individual, as in the case of Walter Snyder.

A second mechanism involves context-free familiarity (Memon, Hope, Bartlett, \& Bull, 2002). By this account, having previously viewed a mugshot causes one to experience a vague feeling of familiarity when one sees that face again, in the absence of recollection of the context in which that face was previously encountered. One may thus mistakenly attribute the familiarity of the face to the crime scene, causing one to believe that the pictured individual must have committed the crime. This mechanism has also received empirical support (e.g., Deffenbacher, Bornstein, \& Penrod, 2006; Haw et al., 2007; Hinz \& Pezdek, 2001; Perfect \& Harris, 2003). Context-free familiarity could explain the false identification of Walter Snyder. In particular, the familiarity associated with Snyder's face stemming from having previously seen his mugshot may have caused the victim to experience a strong feeling of familiarity when she saw him at the police station, causing her to believe that he was the rapist.
A third mechanism involves false recollection of the context in which a pictured face was encountered (Haw et al., 2007). In particular, a mugshot accompanied by a question about a criminal act may cause one to create a specific association between the pictured person and the criminal act. When one later encounters that same face again, this association may cause one to falsely recollect having seen the pictured person commit the criminal act.

Obtaining unequivocal evidence for this third mechanism is difficult because its predictions overlap with those of the other two mechanisms. Haw et al. (2007) tested for false recollection by applying the remember-know-guess procedure (Gardiner \& Richardson-Klavehn, 2000) to a lineup recognition task. Remember judgments were presumed to reflect a phenomenological experience of recollection of one of the target faces, bringing to mind details of the encoding context in which that face was first encountered. They found that participants gave remember judgments not only to target faces seen at encoding but also to faces that had only appeared in an intervening show-up identification task (similar to mugshot identification except that a trial involves a yes-no judgment to just one potential perpetrator). Interpreting this result is difficult, however, because elevated rates of false remember judgments were only made for faces that were incorrectly endorsed during show-up identification. It is thus possible that participants justified their remember responses to these previously encountered faces not on the basis of false recollection of having seen those faces at encoding, but rather on the basis of correct recollection of having previously endorsed those faces during the show-up identification task (i.e., a commitment effect). In contrast, having viewed but not previously endorsed a face during show-up identification led to elevated "know" responses, suggesting an influence of familiarity for the face in the absence of recollection of the context in which that face was encountered (i.e., context-free familiarity).

Empirical evidence that mugshot viewing can lead to false recollection thus remains somewhat equivocal. Findings using related methodologies, however, suggest that such false recollection may occur. For example, Lindsay, Hagen, Read, Wade, and Garry (2004) asked undergraduates to "remember" three childhood events, two of which actually happened and one that did not. Half of the participants were given school class photos to help them remember the events. Although the photos merely depicted participants' former classmates and teachers in stationary poses, viewing those photos led to more reports of "reliving" the imaginary event and to higher confidence that the event had occurred as described, suggesting that participants recollected the event even though it never actually occurred. A possible explanation is that pictures of classmates and teachers, accompanied by stories about what those people had done, caused participants to associate those people with those actions, leading participants to later falsely recollect having seen 
those people perform those actions. If this interpretation is correct, then viewing a mugshot of an individual accompanied by a question about a particular criminal act could lead one to later recollect having seen that person perform that criminal act.

Kersten, Earles, and Upshaw (2013) also found evidence that a target person could become associated with actions that she did not in fact perform, causing eyewitnesses to later falsely recollect having seen the target person perform those actions. Participants viewed a series of brief events, each involving two actors playing two different roles within the event (e.g., one person brushing another person's hair). In a later recognition test, participants saw events in which a target actor performed either the same actions she had performed earlier or actions that had previously been performed by somebody else, either within the same event or in a completely different event. Participants were more likely to falsely recognize a target actor performing actions that had been performed by a different actor within the same event rather than an actor from a different event. Moreover, using a variant of Tulving's (1985) remember-know paradigm, this increased rate of false recognition for recombined actors and actions from the same event was found to be associated with recollection rather than familiarity. This result suggests that the simultaneous presence of an actor and the actions of another person within the same event caused participants to falsely associate that actor and action, causing participants to later falsely recollect having seen that actor perform that action. Although no mugshots were presented in this research, the simultaneous presence of an actor and the actions of another person may have effects similar to those of presenting a picture of a person together with a question about the actions of another person, leading to a spurious association between that person and those actions. This association may later lead to false recollection of having seen that person perform those actions.

In the present research, we examined whether mugshot viewing can lead to false recollection of having seen the pictured actor perform the action in question in a subsequent event recognition test, independently of any effects of commitment or context-free familiarity. The assumption guiding this research is that recollection, familiarity, and commitment reflect the workings of ordinary memory processes, and thus it should not be necessary to expose participants to criminal actions to observe these effects (see Brown, Deffenbacher, \& Sturgill, 1977; Ellis, Shepherd, \& Davies, 1979; Gorenstein \& Ellsworth, 1980; Haw et al., 2007; Hinz \& Pezdek, 2001; Perfect \& Harris, 2003; and Pezdek \& Blandon-Gitlin, 2005, for related approaches). Instead, we presented participants with events involving actors performing everyday actions, using a methodology developed by Kersten, Earles, Curtayne, and Lane (2008; see also Earles, Kersten, Curtayne, \& Perle, 2008; Kersten \& Earles, 2010; Kersten, Earles, \& Berger, 2015). We later presented participants with "mugshots" of various actors accompanied by questions about whether one of the pictured people had performed a particular action. Finally, we tested people on their recognition memory for the events seen earlier. Some of the events in this recognition test were identical to ones seen at encoding, some involved a new actor and action, and others involved a familiar actor performing an action that had previously been performed by somebody else. False recognition of this third type of event would constitute evidence for unconscious transference.

To avoid the ambiguities associated with remember-know judgments in the context of a multiphase experiment, we employed an alternate approach to distinguish familiarity and recollection. In particular, the notion of recollection suggests that a target stimulus has become associated with information about the context in which it was presented, such that later recognition of that stimulus is accompanied by retrieval of contextual information. Thus, if mugshot viewing accompanied by a question about an action leads to a specific association between that person (the target stimulus) and that action (the event context), this could later lead to false recollection of a test event in which that actor performs that action. If this same actor were instead seen at test performing some other familiar action, no such false recollection should occur, because the event context in the test item would not match the contextual information previously associated with that actor. Thus, recollection should lead to an elevated rate of false recognition only for an actor performing the specific action that was suggested when viewing the mugshot of that actor.

In contrast to recollection, familiarity implies a more general feeling that a stimulus has been seen before, in the absence of retrieval of contextual information. Thus, viewing a mugshot of an actor should lead to an elevated rate of false recognition of that actor performing any familiar action. In particular, as long as both the actor and the action were familiar, it should not matter whether they were presented together or separately during mugshot viewing. Thus, participants should be no more likely to falsely recognize an event involving an actor performing the specific action suggested during mugshot viewing than an event involving the actor performing a different familiar action.

Another variable that may be relevant to whether mugshot viewing leads to familiarity or false recollection involves the age of the eyewitness. Older adults have been found to be more likely than young adults to falsely recollect having witnessed an event that was in fact only presented in the form of a static picture (Schacter, Koutstaal, Johnson, Gross, \& Angell, 1997). The information in the picture may have led older adults to mentally simulate the depicted event, causing them to later falsely recollect having witnessed that event. Older adults in the present task may similarly combine information presented in a picture (i.e., an actor's face) with other information accompanying that picture (i.e., a question about an action), leading them to mentally simulate that person 
performing that action. These imagery processes may later lead older adults to falsely recollect having seem that person perform that action.

An alternative prediction, however, derives from NavehBenjamin's (2000) associative-deficit hypothesis. In particular, binding a pictured actor with a suggested action may require associative mechanisms that decline with age. Although this associative deficit is generally viewed as a negative consequence of aging, in this instance it may make older adults less likely to experience false recollection, because they will be less likely to successfully associate a pictured actor with a suggested action. Older adults may thus be more likely than young adults to rely on familiarity, with mugshot exposure leading them to be more likely to falsely recognize any event involving the pictured actor performing a familiar action, even if the mugshot of the actor had accompanied a question about a different action. Young adults, on the other hand, may be more likely to falsely recognize only those events involving an actor performing an action that had accompanied the actor's mugshot, because young adults will be more successful at associating the actor with that specific action.

An issue that must be addressed when comparing young and older adults on their abilities at associating features (e.g., actors and actions) in memory is that the two groups may differ not only in their abilities to associate those features, but also in their abilities to remember each feature in isolation. This makes it difficult to determine whether age differences in the rate of false recognition of novel conjunctions of actors and actions represent a deficit in associative memory, feature memory, or both. Kersten et al. (2008) were able at equate young and older adults on feature memory by using a longer retention interval for young adults. Thus, in the present research, in addition to testing a group of young adults with the same retention interval that was used for older adults, with encoding, mugshot viewing, and recognition all occurring in the same session, a second group of young adults was tested with a three-week interval separating encoding and recognition, just as Kersten et al. had done. These young adults were predicted to perform no better than older adults at remembering the individual features of the events. Young and older adults could thus be compared on their abilities at associating actors and actions in the absence of a young adult advantage in memory for those features in isolation.

To test for influences of mugshot viewing on false recognition of a novel conjunction of a familiar actor and action in young and older adults, participants viewed a series of events involving different actors performing different actions. They were later given verbal questions about which actor had performed each of a number of actions, with each question accompanied by mugshots of potential "perpetrators" of these actions. Finally, participants were tested on their recognition memory for the events, either immediately or three weeks later.
The critical recognition foils in this final recognition memory test were the conjunction items. These items involved a familiar actor performing a familiar action that had been performed by somebody else. Performance with three different types of conjunction items was compared in order to examine the influences of mugshot viewing on false recognition in young and older adults. In one set of conjunction items, the baseline-conjunction items, an actor was seen performing an action that had been performed by a different actor at encoding, but the actor in the conjunction item had not appeared in the mugshot trials (i.e., the actor had only appeared at encoding, performing a different action from the one now being performed). These items provided a baseline for interpreting the influences of mugshot viewing on false recognition of the other two types of conjunction items. In a second set of conjunction items, the consistent-conjunction items, an actor was seen performing an action that had been performed by a different actor at encoding, but a question about this action had later been accompanied by a mugshot of the actor who was now seen performing it. Participants would thus be more likely to falsely recognize consistentconjunction items if mugshot viewing either led to a false association between the pictured actor and the suggested action (i.e., false recollection), or if it simply caused the pictured actor to become more familiar (i.e., context-free familiarity). Finally, in a third set of conjunction items, the inconsistentconjunction items, an actor was seen performing an action that had been performed by a different actor at encoding, but a mugshot of the actor had appeared in relation to a question about a different action from the one that the actor was now seen performing (and different from the one that the actor had performed at encoding). Thus, if mugshot viewing led to a specific association between the pictured actor and the suggested action, this would not lead to increased false recognition of inconsistent-conjunction items, because the action currently being performed was different from the one suggested during mugshot viewing. If, on the other hand, mugshot viewing led to a vague feeling of familiarity for the pictured actor, then participants should still exhibit elevated false recognition of inconsistent-conjunction items, because the actor would indeed have appeared in the mugshot trials (albeit separately from any mention of the action now being performed).

We predicted that older adults would be more likely to falsely recognize either consistent-conjunction or inconsistent-conjunction items than to falsely recognize baseline-conjunction items, providing evidence for older adults' use of familiarity when judging whether an actor performed an action earlier. On the other hand, we predicted that young adults would be more likely to falsely recognize consistent-conjunction items than to falsely recognize either inconsistent- or baseline-conjunction items, providing evidence for false recollection among young adults of having seen the pictured actor perform the suggested action. Finally, 
we predicted that these patterns would still hold even when the analysis was limited to items that participants had not endorsed in the mugshot trials, providing evidence that these effects occur independently of commitment on the part of young and older adults.

\section{Method}

\section{Participants}

Eighty undergraduates at Florida Atlantic University (mean age $=19.33, S D=1.09)$ received course credit for participation. Forty older adults (mean age $=71.76, S D=8.01$ ) received \$20 gift certificates. An a priori power analysis conducted using G*Power 3 (Faul, Erdfelder, Lang, \& Buchner, 2007 ) indicated that 120 participants would be needed to detect an interaction of participant group with the critical contrast on item type (i.e., consistent conjunction vs. inconsistent conjunction), assuming an effect size of .2 and a correlation of .4 among repeated measures. The participants' characteristics are given in Table 1 .

\section{Stimuli}

Ten lists of 36 encoding items were created using the videos filmed by Kersten et al. (2008). Each video involved a female actor seated at a desk, performing a simple action such as watering a plant or lighting a flashlight. The first two and last two videos were identical for all participants, serving as primacy and recency fillers. The remaining 32 items were presented in a unique random order for each participant.

Eighty-four "mugshots" were created by capturing individual frames from the encoding videos. Each frame was cropped to include just the head and shoulders of the actor, and was rescaled to $10 \mathrm{~cm} \times 10 \mathrm{~cm}$ in size. Each mugshot trial involved two mugshots, one depicting the target actor and one depicting a new actor, randomly chosen from the set of actors not seen by a given participant. Which actor appeared on the left and which appeared on the right was determined randomly for each trial. Each pair of mugshots was accompanied by a question about a particular action (e.g., "Which of these people did you see watering a plant?"), with buttons labeled "Person 1" and "Person 2" appearing below the two pictures, and a button labeled "Neither Person" appearing farther below. The first two and last two items tested memory for the actions seen in the encoding filler items, with the actors who had performed those actions now serving as the target actors. The remaining 32 items were presented in a unique random order for each participant. In the eight old items, the target actor had indeed performed the action in question. In the 16 conjunction items, the target actor had performed a different action from the one in question. Finally, in the eight new-actor items, the target
Table 1 Participant characteristics

\begin{tabular}{|c|c|c|c|c|}
\hline & \multicolumn{2}{|c|}{ Young Adults } & \multicolumn{2}{|c|}{ Older Adults } \\
\hline & $M$ & $(S D)$ & $M$ & $(S D)$ \\
\hline Age & 19.33 & (1.09) & 71.76 & (8.01) \\
\hline Health & 3.95 & $(0.81)$ & 4.26 & $(0.85)$ \\
\hline Medications & 0.29 & $(0.60)$ & 3.55 & (2.31) \\
\hline Education & 12.49 & $(0.90)$ & 15.78 & (3.11) \\
\hline Vocabulary & 27.61 & (3.90) & 37.28 & (1.55) \\
\hline
\end{tabular}

Health $=$ self-reported health on a scale of 1 (poor) to 5 (excellent). Medications $=$ number of prescription medications taken. Education $=$ years of education, with 12 indicating completion of high school and 16 indicating completion of college. Vocabulary $=$ score out of 40 vocabulary items from the Shipley (1986) vocabulary test.

actor had not been seen before by the participant, and thus both the actors appearing in these items were new.

Ten lists of 44 event recognition items were created using the videos filmed by Kersten et al. (2008). A unique random order of recognition items was used for each participant. Four filler items involved an actor from the encoding filler items performing the same action she had performed previously. Eight old items involved an actor performing the same action she had performed at encoding. A mugshot of that actor had also accompanied a question about that action. Eight new items involved an actor not seen either at encoding or in the mugshot trials, performing an action not seen at encoding or mentioned in the mugshot trials. Twenty-four conjunction items involved an actor seen at encoding performing an action that had been performed by somebody else at encoding. Of these, eight baseline-conjunction items involved an actor who had not appeared in the mugshot trials, with a question about the action she was now seen performing having instead been accompanied by mugshots of new actors. Eight consistentconjunction items involved an actor whose mugshot had appeared along with a question about the action that she was now seen performing (but different from the one she had performed at encoding). Finally, eight inconsistent-conjunction items involved an actor whose mugshot had appeared along with a question about an action that was different from the one she was now seen performing (and also different from the one she had performed at encoding), with a question about the action she was now seen performing having instead been accompanied by a mugshot of another familiar actor.

\section{Procedure}

The participants were instructed to remember which person had performed each action. They then viewed 36 encoding items, clicking a button labeled "Next Event" after each event to continue. Next they completed a demographic questionnaire, followed by 40 items from the Shipley (1986) 
vocabulary test. Following this, participants received 36 mugshot trials. After they had clicked on the button underneath one of the actors (or on the "Neither Person" button), they rated their confidence by clicking on one of three buttons, labeled "absolutely sure," "pretty sure," and "just guessing."

After completing the mugshot trials, older adults and half of the young adults were immediately given the event recognition trials, whereas the other young adults returned three weeks later. Participants were instructed that an actor would sometimes be seen performing an action that had been performed by somebody else, and that they should respond "no" to these items. They were also instructed that they would sometimes see an actor performing an action that she had not previously performed but that had been mentioned in relation to her picture in the mugshot trials. They were also instructed to respond "no" to these items. After each video, participants were asked "Did you see this person perform this action in the first part of the experiment?" After clicking on one of two buttons labeled "Yes" and "No," participants rated their confidence using the same rating scale as in the mugshot trials.

\section{Results}

\section{Mugshot trials}

The results of the mugshot trials are presented in Table 2 . Preliminary analyses comparing young adults in the immediate and delayed testing conditions revealed no significant differences, and thus the two groups were combined in subsequent analyses of performance in the mugshot trials. An analysis of variance (ANOVA) on the proportions of target faces selected, with age group (young vs. older adult) and item type (old vs. conjunction vs. new actor) as independent variables, revealed a main effect of item type, $F(2,236)=140.74, M S E=$ $.023, p<.001, \eta_{\mathrm{p}}{ }^{2}=.54$, as well as an interaction of item type with age group, $F(2,236)=10.61, M S E=.023, p<.001, \eta_{\mathrm{p}}{ }^{2}=$ .08 . To understand this interaction, the two age groups were compared on each of the three item types. Young adults were found to be more likely than older adults to correctly recognize old mugshots, $t(118)=3.29, p=.001, d=0.61$, whereas older adults were more likely than young adults to falsely recognize conjunction mugshots, $t(118)=2.73, p=.007, d$ $=0.50$. The two groups were equally likely to falsely recognize new-actor mugshots, $t(118)=0.14, p>.10, d=0.03$. Young adults were thus more successful than older adults at associating a familiar face with the correct action.

\section{Event recognition}

An ANOVA was conducted on the proportions of "yes" responses, with participant group and item type as independent variables. This analysis revealed a main effect of participant group, $F(2,117)=6.12, M S E=.013, p=.003, \eta_{\mathrm{p}}{ }^{2}=.10$, with older adults exhibiting a higher overall rate of "yes" responses than either of the young-adult groups. The effects of item type were analyzed using four orthogonal planned comparisons (see Table 3). The relevant means for the first two comparisons are presented in Fig. 1. The first comparison, contrasting old items with all other item types, was significant, $F(1,117)=$ $876.11, M S E=.037, p<.001, \eta_{\mathrm{p}}{ }^{2}=.88$. This comparison also showed an interaction with participant group, $F(2,117)=$ $32.53, M S E=.037, p<.001, \eta_{\mathrm{p}}{ }^{2}=.36$, indicating significant differences between the three groups in terms of discriminating old items from recognition foils. Young adults with no delay outperformed older adults, $F(1,78)=32.12, M S E=$ $.033, p<.001, \eta_{\mathrm{p}}{ }^{2}=.29$, whereas older adults outperformed

Table 2 Proportions of different types of choices in the mugshot trials

\begin{tabular}{|c|c|c|c|c|c|c|c|}
\hline \multirow[t]{2}{*}{ Participant Group } & \multirow[t]{2}{*}{ Item Type } & \multicolumn{2}{|c|}{ Target Photo } & \multicolumn{2}{|c|}{ Nontarget Photo } & \multicolumn{2}{|c|}{ Neither Photo } \\
\hline & & $M$ & $(S D)$ & $M$ & $(S D)$ & $M$ & $(S D)$ \\
\hline \multirow[t]{3}{*}{ Young, No Delay } & Old & .66 & $(.18)$ & .15 & $(.14)$ & .19 & $(.15)$ \\
\hline & Conjunction & .23 & $(.12)$ & .24 & $(.15)$ & .53 & $(.19)$ \\
\hline & New actor & .24 & $(.16)$ & .21 & $(.15)$ & .55 & $(.24)$ \\
\hline \multirow[t]{3}{*}{ Older Adults } & Old & .51 & $(.18)$ & .19 & $(.17)$ & .30 & $(.21)$ \\
\hline & Conjunction & .32 & $(.15)$ & .23 & $(.12)$ & .45 & $(.21)$ \\
\hline & New actor & .25 & $(.16)$ & .23 & (.14) & .52 & $(.23)$ \\
\hline \multirow[t]{3}{*}{ Young, 3-Week Delay } & Old & .59 & $(.20)$ & .14 & $(.14)$ & .27 & $(.18)$ \\
\hline & Conjunction & .27 & $(.12)$ & .23 & (.13) & .50 & $(.18)$ \\
\hline & New actor & .25 & (.19) & .24 & (.16) & .51 & $(.25)$ \\
\hline
\end{tabular}

The target photos in the old trials were in fact photos of the actor who had performed the action in question, whereas the nontarget photos were of a new actor. The target photos in the conjunction trials were photos of a familiar actor who had been seen doing something else, whereas the nontarget photos were of a new actor. The target photos in the new-actor items were of an unfamiliar actor, just as were the nontarget photos. The three mean proportions in each row add up to 1.0 because they represent the three possible answers to each item. 
Table 3 Contrast weights for the orthogonal planned comparisons involving item type

\begin{tabular}{lllll}
\hline Item Type & \multicolumn{2}{l}{ Contrast } & & \\
\cline { 2 - 5 } & 1 & 2 & 3 & 4 \\
\hline Old & 1 & 0 & 0 & 0 \\
Consistent conjunction & $-1 / 4$ & $1 / 3$ & $1 / 2$ & 1 \\
Inconsistent conjunction & $-1 / 4$ & $1 / 3$ & $1 / 2$ & -1 \\
Baseline conjunction & $-1 / 4$ & $1 / 3$ & -1 & 0 \\
New & $-1 / 4$ & -1 & 0 & 0
\end{tabular}

The first comparison examined discrimination of old items from recognition foils, contrasting correct "yes" responses to old items with incorrect "yes" responses to the other item types. The second comparison examined the effects of the familiarity of the actor and action on false recognition of foils, contrasting "yes" responses to new items (in which the actor and action were unfamiliar) with "yes" responses to the three types of conjunction items (in which the actor and action were familiar). The third comparison examined the effects of mugshot presentation on false recognition of conjunction items, contrasting "yes" responses to baselineconjunction items with "yes" responses to consistent-conjunction and inconsistent-conjunction items. Finally, the fourth comparison examined whether mugshot presentation resulted in the creation of a specific association between the pictured actor and the action in question, contrasting "yes" responses to consistent-conjunction items with yes" responses to inconsistent-conjunction items.

young adults with a three-week delay, $F(1,78)=5.63, M S E=$ $.043, p=.02, \eta_{\mathrm{p}}{ }^{2}=.07$. Of particular interest is that older adults performed significantly better than young adults with a three-week delay at discriminating old items from new items involving new actors and actions, $t(78)=4.52, p<.001, d=$ 1.01. This suggests that imposing a three-week delay on young adults led to feature memory performance that was below that of older adults, thus allowing us to examine the abilities of the two participant groups to associate those

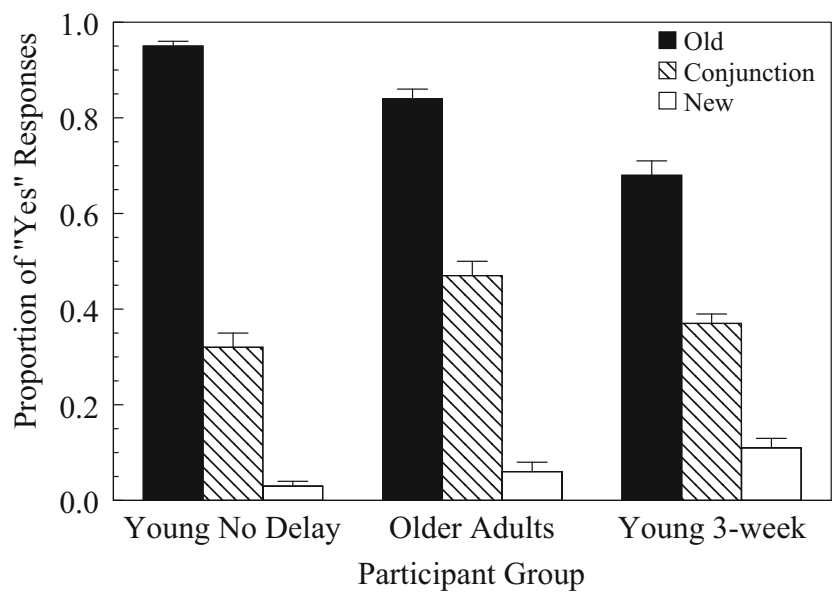

Fig. 1 Proportions of "yes" responses to the old, conjunction, and new items by the three participant groups, with the three types of conjunction items aggregated together into a single category. "Yes" responses to the old items were correct, whereas "yes" responses to the conjunction and new items constituted errors. Error bars represent the standard errors of the means. features in the absence of a young-adult advantage in memory for the features themselves.

The second comparison, contrasting new items with conjunction items, was also significant, $F(1,117)=443.72, M S E$ $=.028, p<.001, \eta_{\mathrm{p}}^{2}=.79$. This comparison also revealed an interaction with participant group, $F(2,117)=8.74, M S E=$ $.028, p<.001, \eta_{\mathrm{p}}{ }^{2}=.13$, indicating significant differences between the three groups in the extents to which they were influenced by the familiarity of the actors and actions in the conjunction items. Older adults exhibited greater influences of familiarity than did either young adults with no delay, $F(1,78)$ $=10.02, M S E=.030, p=.002, \eta_{\mathrm{p}}^{2}=.11$, or young adults with a three-week delay, $F(1,78)=14.27, M S E=.029, p<$ $.001, \eta_{\mathrm{p}}{ }^{2}=.16$. We observed no significant difference between the two young-adult groups, $F(1,78)<1$.

The relevant means for the third and fourth comparisons, which contrasted the three different types of conjunction items, are presented in Fig. 2. The third comparison, contrasting baseline conjunction items with both consistent- and inconsistent-conjunction items, was significant, $F(1,117)=$ $8.08, M S E=.048, p=.005, \eta_{\mathrm{p}}{ }^{2}=.07$. This comparison did not interact with participant group, $F(2,117)<1$, indicating that the three participant groups did not differ in the overall extents to which they were influenced by mugshot presentations of the actors.

Finally, the fourth comparison, contrasting consistentconjunction and inconsistent-conjunction items, was significant, $F(1,117)=4.05, M S E=.049, p=.05, \eta_{\mathrm{p}}{ }^{2}=.03$. This comparison interacted with participant group, $F(2,117)=$ $3.55, M S E=.049, p=.03, \eta_{\mathrm{p}}{ }^{2}=.06$, indicating significant differences between the three groups in the extents to which they specifically associated the actor appearing in a mugshot with the action mentioned in relation to that mugshot. Young adults with a three-week delay exhibited significantly greater discrimination of consistent- and inconsistent-conjunction

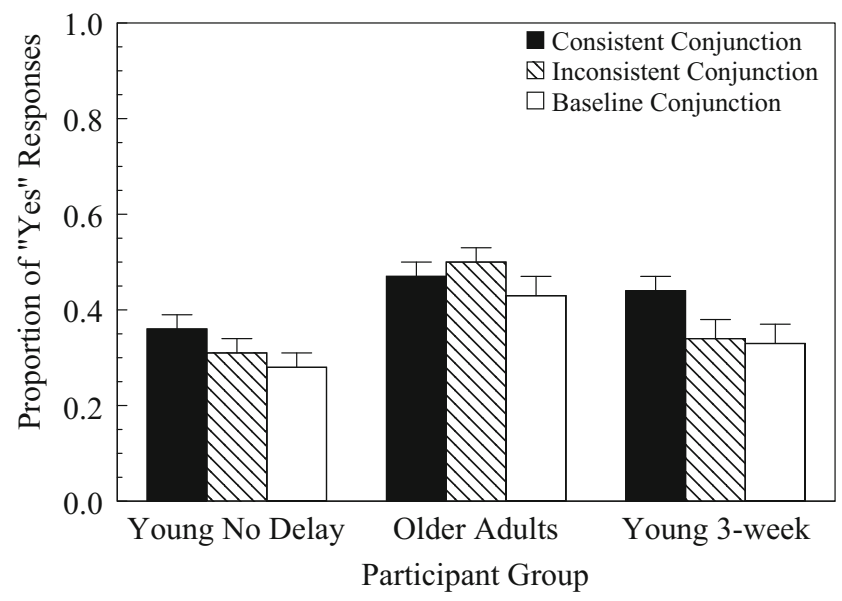

Fig. 2 Proportions of incorrect "yes" responses to the three different types of conjunction items by the three participant groups. Error bars represent the standard errors of the means. 
items than did older adults, $F(1,78)=6.91, M S E=.050, p=$ $.01, \eta_{\mathrm{p}}{ }^{2}=.08$, and this same comparison also approached significance when comparing young adults with no delay to older adults, $F(1,78)=2.78, M S E=.041, p=.10, \eta_{\mathrm{p}}{ }^{2}=.03$. No significant difference emerged between the two youngadult groups, $F(1,78)<1.13$. Combining the two youngadult groups, young adults were more likely to falsely recognize the consistent-conjunction than the inconsistentconjunction items, $t(79)=2.82, p=.006, d=0.35$, whereas there was a nonsignificant trend in the opposite direction for older adults, $t(39)=-0.96, p>.10, d=-0.14$.

In summary, although all three participant groups were more likely to falsely recognize conjunction items if the actors appearing in those items had also appeared in the mugshots, the mechanisms underlying this effect appear to be different for young and older adults. Older adults were more likely to falsely recognize a conjunction item if the actor had previously appeared in a mugshot, regardless of which action had been mentioned in relation to that mugshot. Young adults, on the other hand, were more likely to falsely recognize a conjunction item if a mugshot of the actor had been accompanied by a question about the action that the actor was now seen performing. This suggests that young adults formed a specific association between the pictured actor and the queried action, causing them to later falsely recollect having seen that actor perform that action.

\section{Contingencies between mugshot identification and event recognition}

A possible alternative explanation for young adults' higher rate of false recognition of consistent-conjunction than of inconsistent-conjunction items is that they remembered which face they had chosen in each mugshot trial and responded to the event recognition trials in a manner that was consistent with those earlier choices (i.e., a commitment effect). Because the actor in a consistent-conjunction item had appeared in the corresponding mugshot trial, whereas the actor in an inconsistent-conjunction item had not, this may have made participants more likely to accept the consistentconjunction items than to accept the inconsistent-conjunction items. To test whether performance with the consistentconjunction items was driven in part by commitment to prior selections in the mugshot trials, the consistent-conjunction items were divided into those in which the target actor's face had previously been selected in the mugshot identification trials, those in which the other actor who had appeared with this actor had been selected, and those in which neither actor had been selected. An ANOVA was then conducted on the proportions of trials of each type that were falsely recognized by each participant. The two young-adult groups were collapsed in this and subsequent analyses because their performance with the conjunction items did not significantly differ in the primary analysis. Thirty-nine participants (24 young and 15 old) were excluded from this analysis because they did not have at least one trial of each type.

The results of the remaining participants are depicted in Fig. 3. Analysis of these results revealed a main effect of prior selection, $F(2,158)=9.56, M S E=.104, p<.001, \eta_{\mathrm{p}}{ }^{2}=.11$. Post-hoc $t$ tests (with Bonferroni adjustment for multiple comparisons) revealed that participants were more likely to falsely recognize consistent-conjunction items when they had previously selected the same actor in the mugshot trials, as compared to both when they had selected the other actor in those trials, $t(80)=4.00, p<.001, d=0.45$, and when they selected neither actor in those trials, $t(80)=4.35, p<.001, d=0.49$. We did not find a significant difference between trials in which participants had previously selected the other actor and trials in which they had selected neither actor, $t(80)=0.23, p>.10$, $d=0.03$. No significant effects involved participant group, both $F \mathrm{~s}<1$, indicating that both age groups were more likely to falsely recognize consistent-conjunction items involving actors they had previously selected as having performed the same actions in the mugshot trials.

The previous analysis suggests that false recognition of at least some of the consistent-conjunction items may have reflected commitment effects. To determine whether commitment effects entirely account for young adults' higher rates of false recognition of consistent- than of inconsistentconjunction items, or whether some other mechanism (e.g., false recollection) contributes to this difference, performance with these two types of items was compared under conditions

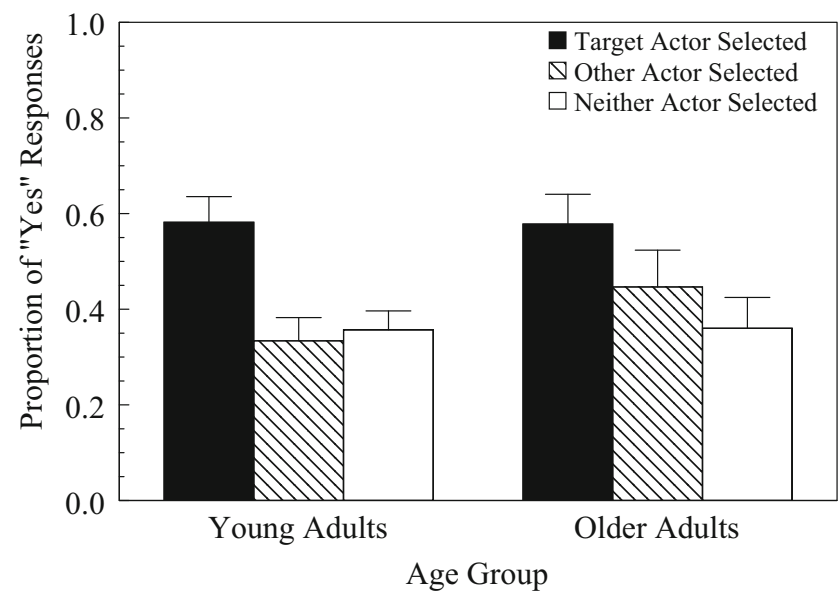

Fig. 3 Proportions of incorrect "yes" responses to consistent-conjunction items in relation to participants' earlier selections in the mugshot trials. Target Actor Selected indicates that the actor performing the action in a recognition item had been previously selected in the mugshot trials as having performed that action. Other Actor Selected indicates that the actor performing the action in a recognition item had not been previously selected in the mugshot trials, but rather, the other actor who had appeared with this actor had been selected. Neither Actor Selected indicates that the actor performing the action in a recognition item had not been previously selected in the mugshot trials, but rather, neither actor had been selected. Error bars represent the standard errors of the means. 
in which no prior commitment had been made. In particular, the analysis was limited to consistent- and inconsistentconjunction items involving actions for which neither actor had been selected in the mugshot identification trials, and involving actors that had not been selected as having performed any action in the mugshot identification trials. If the difference in the rates of false recognition of the consistentand inconsistent-conjunction items solely reflects a commitment effect, this difference should then disappear when only considering items for which participants did not commit to either actor as having performed the action in question, and did not commit to the actor appearing in those items as having performed some other action. If, on the other hand, it in part reflects false recollection of having seen an actor perform an action, resulting from the pairing of that actor and action in the mugshot trials, then a higher rate of false recognition in the consistent-conjunction trials than in the inconsistentconjunction trials should still be evident, even when neither face was ultimately selected in the mugshot trials.

Seventy-three young adults and 30 older adults had at least one consistent-conjunction trial and at least one inconsistentconjunction trial for which no prior commitment had been made in the mugshot trials. The results of these participants are depicted in Fig. 4. Consistent with the primary analysis, young adults continued to exhibit a higher rate of false recognition of the consistent-conjunction than of the inconsistentconjunction items, $t(72)=2.33, p=.02, d=0.34$, whereas there was a nonsignificant trend in the opposite direction for older adults, $t(29)=-0.83, p>.10, d=-0.19$. These results suggest that commitment effects cannot explain young adults' higher rate of false recognition of the consistent-conjunction than of the inconsistent-conjunction items. This difference may instead reflect false recollection of having seen an actor perform a particular action, stemming from prior mugshot

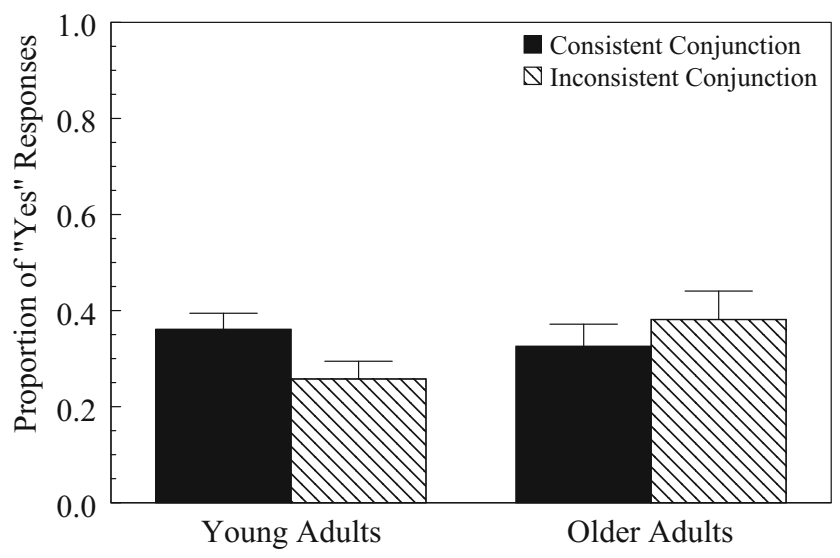

Age Group

Fig. 4 Proportions of incorrect "yes" responses to consistent-conjunction and inconsistent-conjunction items involving actions for which neither actor had been selected in the mugshot trials, and involving actors that had not previously been selected as having performed some other action. Error bars represent the standard errors of the means. viewing in which the actor's photo accompanied a question about that action, regardless of whether participants had actually selected the actor at that time.

\section{Discussion}

This research demonstrated that viewing a mugshot along with a question about an action can lead to the creation of a specific association between the pictured person and the queried action. This association might lead young adults to later falsely recollect having seen the pictured person perform the queried action. It also demonstrates that mugshot viewing can lead older eyewitnesses to experience a feeling of familiarity when considering the combination of the pictured person and a familiar action, in the absence of recollection of the sources of that familiarity.

\section{Mechanisms underlying the effects of mugshot viewing on later recognition}

The performance of different participant groups provides evidence for all three of the mechanisms described in the introduction regarding the effects of mugshot viewing on later recognition. Evidence for false recollection comes from the performance of both groups of young adults. These participants were more likely to falsely recognize an event involving an actor performing an action that had actually been performed by somebody else if they had seen a mugshot of that actor accompanied by a question about that action. This result suggests that mugshot presentation together with a question about an action leads to the creation of a specific association between the pictured actor and the queried action, leading to later false recollection of having seen that actor perform that action.

Interestingly, this effect occurred even when participants did not select the pictured actor as having performed the action in question during mugshot viewing. This suggests that the act of considering whether a given person had performed a particular action may have led participants to form mental images of what the event would have looked like with that person performing the action, even if the participant ultimately decided that the person was not the one who performed that action. These images may have later triggered a phenomenological experience of recollection when the participant was actually presented with a video of that person performing the action, causing the participant to believe that he or she had seen that person perform that same action earlier. Mugshot viewing may thus have functioned similarly to viewing class photos in the research of Lindsay et al. (2004), providing detailed perceptual information about the appearance of an actor that could be combined with mental imagery for an event, creating 
a compelling false memory for having seen that actor participate in that event.

Evidence for a second mechanism, context-free familiarity, comes from the performance of older adults. These participants were more likely to falsely recognize an event involving an actor performing an action that had actually been performed by somebody else if they had seen a picture of that actor during mugshot viewing, regardless of whether the picture had accompanied a question about that action or some other action. This result suggests that mugshot viewing led older adults to experience a strong feeling of familiarity when later viewing the pictured actor performing any familiar action. This global feeling of familiarity may have led older adults to believe that they must have seen this actor perform this same action earlier. This feeling of familiarity must have occurred in the absence of correct recollection of the actual action that the actor had performed at encoding, as well as in the absence of false recollection of having seen the actor perform the action suggested during mugshot viewing, either of which would have encouraged older adults to reject an event involving an actor performing a third action that was different from both of these earlier actions. We found no evidence for increased levels of false recollection associated with aging in the present results, with young adults instead exhibiting higher levels of false recollection than older adults. This may have been because false recollection in the present study involved the creation of a specific association between a pictured actor and a suggested action, and thus required associative mechanisms that decline with age. The present results with older adults are thus consistent with the theory of context-free familiarity stemming from mugshot viewing, as suggested by Memon et al. (2002), as well as an agerelated associative deficit, as was suggested by NavehBenjamin (2000).

There was also evidence for commitment effects in the present results, although the mechanisms underlying these effects may not be completely independent from the mechanisms underlying the false recollection and context-free familiarity effects described above. In particular, all three participant groups were more likely to falsely remember having seen a given actor perform an action that had actually been performed by somebody else if they had selected that actor's photo during the mugshot identification trials. It is especially interesting that older adults exhibited this pattern, even though they were no more likely to falsely recognize consistentconjunction items than to falsely recognize inconsistentconjunction items. If older adults remembered having selected a particular actor's photo in a mugshot trial regarding a particular action, this should have made them more likely to later falsely recognize an item involving that actor performing that action (i.e., a consistent-conjunction item) than to falsely recognize an item involving that actor performing some other action (i.e., an inconsistent-conjunction item).
One possible explanation for this pattern of performance in older adults is that these apparent commitment effects simply represent another manifestation of contextfree familiarity. In particular, when presented with a mugshot trial involving an actor who had been seen doing something other than the action in question, along with an entirely new actor, choosing the familiar (but still incorrect) actor may indicate that the participant received a feeling of familiarity for the actor, but was unable to recollect the action that the actor had previously performed. When later presented with an event involving that same actor performing either the action queried during the mugshot trials or some other action, they would have received a powerful feeling of familiarity for the actor, stemming from both the encoding event in which she had appeared and the later mugshot trial, perhaps making them more likely to falsely recognize that event. Commitment effects may thus serve as an indicator of the degree to which participants experienced feelings of familiarity for the actors in the conjunction trials.

It may also be possible to explain commitment effects in terms of recollection, however, if one assumes that recollection may be partial rather than all or none (see, e.g., Wixted \& Mickes, 2010). In particular, participants may have recollected seeing a particular actor during mugshot viewing and selecting that actor, but not necessarily remembering the action for which they had endorsed that actor. This recollection of having previously selected the actor may have made them more likely to later accept the combination of that actor and any familiar action. By this account, recollection of which question had accompanied a particular face and recollection of their response to that face may have occurred somewhat independently, with the former leading to greater acceptance of consistent-conjunction over the inconsistent-conjunction items and the latter leading to greater acceptance of previously selected over previously unselected faces.

This account would allow one to reconcile the present results with those of Haw et al. (2007), who found that commitment effects were evident in remember judgments rather than know judgments. This result suggests that their participants were experiencing recollection when they incorrectly selected faces that had not been present at encoding, but rather had only appeared in an intervening show-up identification task. The basis for these remember judgments, however, may have been the recollection of having previously endorsed these faces in the show-up identification task, rather than the recollection of the circumstances under which the faces were encountered. Commitment effects in the present research may similarly involve recollection of a participant's prior response to a given face, possibly in the absence of recollection of the context in which the face was encountered (e.g., the question that had accompanied it). 


\section{Implications for eyewitness testimony}

The present findings add to a growing body of research demonstrating that mugshot viewing can contaminate eyewitness memory for an event, possibly causing the eyewitness to remember having seen a different person perpetrate the actions in question. We thus concur with other researchers (e.g., Dysart et al., 2001; Goodsell et al., 2009; Haw et al., 2007; Memon et al., 2002) that although mugshot viewing can be a useful investigative technique for identifying and apprehending a criminal, after viewing those mugshots an eyewitness should not be asked to make further identifications as part of the effort to obtain a conviction. Thus, if there are multiple eyewitnesses to a crime, only some of those should be exposed to mugshots during investigative procedures so that others can make a later identification in the absence of contamination from this prior viewing. If there is only one witness to a crime, then the witness will likely be asked to help in identifying the criminal in the early stages of the investigation, leaving open the possibility of contamination of the witness' memory for the crime. After apprehending the perpetrator in these circumstances, other forensic evidence should ideally be used to corroborate the testimony of the witness, given this possibility of contamination of eyewitness memory.

The finding that mugshot viewing can lead to a specific association between the pictured person and the action in question is particularly important because such specific associations may later give rise to a recollective experience of having seen the pictured person perform the queried action. False recollection is especially problematic from a legal perspective because recollection has been found to be associated with the highest levels of confidence on the part of observers (Yonelinas \& Parks, 2007), and a confident eyewitness may have a particularly powerful influence on a jury during courtroom deliberations. Thus, if mugshot viewing leads to false recollection in eyewitnesses to real-world crimes, this may lead witnesses to attribute high levels of confidence to their memories, making them particularly compelling on the witness stand.

\section{Caveats}

Of course, the present experimental methodology differs from the real-world experience of an eyewitness in important ways that may affect the generalizability of the present results to actual criminal cases. Most notably, participants were not presented with emotionally laden criminal acts, but rather with simple everyday actions. It is thus possible that the distinctiveness of criminal actions makes eyewitnesses less likely to confuse the perpetrators of such actions with the perpetrators of ordinary actions. The growing number of DNA exonerations of innocent people who were convicted on the basis of false eyewitness testimony, however, suggests that eyewitnesses to actual crimes are not immune to influences of the false memory mechanisms documented in this research. Indeed, recent research by Earles, Kersten, Vernon, and Starkings (2016) suggests that emotional content may sometimes lead to increased rather than reduced likelihood of later falsely remembering having seen an action carried out by someone who had actually been seen doing something else. Moreover, a great deal of eyewitness testimony focuses not on emotional criminal acts but rather on ordinary types of actions (e.g., who was talking to whom) that take on added importance in the context of the legal system. Thus, although one must be cautious in generalizing the present results to eyewitness memory for violent crimes, they may still straightforwardly apply to the large body of eyewitness testimony centering on everyday types of actions.

A second caveat is that participants were tested on their memory for many actions performed by many actors, whereas real-world eyewitnesses are typically questioned about their memory for only a small number of actions. Testing for such a large number of actions no doubt increases the possibility for confusions in memory, especially compared to a situation in which memory is being tested for a criminal, emotionally laden action that is distinct from the milieu of ordinary actions that one observes on a daily basis. Still, given that people do encounter many people performing many actions in everyday life, the possibility for confusion in memory for the sources of those actions remains, especially for actions that are not as distinctive as a violent crime but are still often the focus of eyewitness testimony.

In closing, although it is difficult to generalize from laboratory findings to real-world cases such as the one described earlier, the present results suggest that the powerful feeling of dread that the victim experienced upon seeing Walter Snyder in the lobby of the police station may have stemmed from a specific association between his face and the crime in which she had been victimized. This association may have formed as a result of viewing a mugshot of his face and considering whether he had committed the crime. The recollective experience of the victim resulting from the retrieval of this association may have made her extremely confident that he was in fact the perpetrator, with her ensuing, highly compelling testimony ultimately leading to his conviction, largely in the absence of supporting evidence. As a result of this error in eyewitness testimony, Snyder served seven years in prison for a crime he did not commit, whereas the identity of the actual perpetrator will likely never be known. 
Author note This research was supported by National Institute on Aging Grant No. R15-AG23526.

\section{References}

Blunt, M. R., \& McAllister, H. A. (2009). Mug shot exposure effects: Does size matter? Law and Human Behavior, 33, 175-182. doi:10.1007/s10979-008-9126-z

Brigham, J. C., \& Cairns, D. L. (1988). The effect of mugshot inspections on eyewitness identification accuracy. Journal of Applied Social Psychology, 18, 1394-1410. doi:10.1111/j.1559-1816.1988. tb01214.x

Brown, E., Deffenbacher, K., \& Sturgill, W. (1977). Memory for faces and the circumstances of encounter. Journal of Applied Psychology, 62, 311-318. doi:10.1037/0021-9010.62.3.311

Deffenbacher, K. A., Bornstein, B. H., \& Penrod, S. D. (2006). Mugshot exposure effects: Retroactive interference, mugshot commitment, source confusion, and unconscious transference. Law and Human Behavior, 30, 287-307. doi:10.1007/s10979-006-9008-1

Dysart, J. E., Lindsay, R. C. L., Hammond, R., \& Dupuis, P. (2001). Mug shot exposure prior to lineup identification: Interference, transference, and commitment effects. Journal of Applied Psychology, 86, 1280-1284. doi:10.1037/0021-9010.86.6.1280

Earles, J. L., Kersten, A. W., Curtayne, E. S., \& Perle, J. G. (2008). That's the man who did it, or was it a woman? Actor similarity and binding errors in event memory. Psychonomic Bulletin \& Review, 15, 11851189. doi:10.3758/PBR.15.6.1185

Earles, J. L., Kersten, A. W., Vernon, L. L., \& Starkings, R. (2016). Memory for positive, negative, and neutral events in younger and older adults: Does emotion influence binding in event memory? Cognition and Emotion, 30, 378-388. doi:10.1080 02699931.2014.996530

Ellis, H. D., Shepherd, J. W., \& Davies, G. M. (1979). Identification of familiar and unfamiliar faces from internal and external features: Some implications for theories of face recognition. Perception, 8 , 431-439.

Faul, F., Erdfelder, E., Lang, A.-G., \& Buchner, A. (2007). G*Power 3: A flexible statistical power analysis program for the social, behavioral, and biomedical sciences. Behavior Research Methods, 39, 175-191. doi:10.3758/BF03193146

Gardiner, J. M., \& Richardson-Klavehn, A. (2000). Remembering and knowing. In E. E. Tulving \& F. I. M. Craik (Eds.), The Oxford handbook of memory (pp. 229-244). New York, NY: Oxford University Press.

Goodsell, C. A., Gronlund, S. D., \& Neuschatz, J. S. (2015). Investigating mug shot commitment. Psychology Crime and Law, 21, 219-233. doi:10.1080/1068316X.2014.951647

Goodsell, C. A., Neuschatz, J. S., \& Gronlund, S. D. (2009). Effects of mugshot commitment on lineup performance in young and older adults. Applied Cognitive Psychology, 23, 788-803. doi:10.1002 /acp. 1512

Gorenstein, G. W., \& Ellsworth, P. C. (1980). Effect of choosing an incorrect photograph on a later identification by an eyewitness. Journal of Applied Psychology, 65, 616-622. doi:10.1037/00219010.65.5.616

Haw, R. M., Dickinson, J. J., \& Meissner, C. A. (2007). The phenomenology of carryover effects between show-up and line-up identification. Memory, 15, 117-127. doi:10.1080/09658210601171672
Hinz, T., \& Pezdek, K. (2001). The effect of exposure to multiple lineups on face identification accuracy. Law and Human Behavior, 25, 185 198. doi:10.1023/A:1005697431830

Kersten, A. W., \& Earles, J. L. (2010). Effects of aging, distraction, and response pressure on the binding of actors and actions. Psychology and Aging, 25, 620-630. doi:10.1037/a0019131

Kersten, A. W., Earles, J. L., \& Berger, J. D. (2015). Recollection and unitization in associating actors with extrinsic and intrinsic motions. Journal of Experimental Psychology: General, 144, 274-298. doi:10.1037/a0038809

Kersten, A. W., Earles, J. L., Curtayne, E. S., \& Lane, J. C. (2008). Adult age differences in binding actors and actions in memory for events. Memory \& Cognition, 36, 119-131. doi:10.3758/MC.1.119

Kersten, A. W., Earles, J. L., \& Upshaw, C. (2013). False recollection of the role played by an actor in an event. Memory \& Cognition, 41, 1144-1158. doi:10.3758/s13421-013-0334-5

Lindsay, D. S., Hagen, L., Read, J. D., Wade, K. A., \& Garry, M. (2004). True photographs and false memories. Psychological Science, 15, 149-154. doi:10.1111/j.0956-7976.2004.01503002.x

Lindsay, R. C. L., Nosworthy, G. J., Martin, R., \& Martynuck, C. (1994). Using mug shots to find suspects. Journal of Applied Psychology, 79, 121-130. doi:10.1037/0021-9010.79.1.121

Loftus, E. F. (1976). Unconscious transference in eyewitness identification. Law and Psychology Review, 2, 93-98.

Memon, A., Hope, L., Bartlett, J., \& Bull, R. (2002). Eyewitness recognition errors: The effects of mugshot viewing and choosing in young and older adults. Memory \& Cognition, 30, 1219-1227. doi:10.3758 /BF03213404

Naveh-Benjamin, M. (2000). Adult age differences in memory performance: Tests of an associative deficit hypothesis. Journal of Experimental Psychology: Learning, Memory, and Cognition, 26, 1170-1187. doi:10.1037/0278-7393.26.5.1170

Perfect, T. J., \& Harris, L. J. (2003). Adult age differences in unconscious transference: Source confusion or identity blending? Memory \& Cognition, 31, 570-580. doi:10.3758/BF03196098

Pezdek, K., \& Blandon-Gitlin, I. (2005). When is an intervening line-up most likely to affect eyewitness identification accuracy? Legal and Criminological Psychology, 10, 247-263. doi:10.1348/135532505 X49846

Schacter, D. L., Koutstaal, W., Johnson, M. K., Gross, M. A., \& Angell, K. E. (1997). False recollection induced by photographs: A comparison of older and younger adults. Psychology and Aging, 12, 203 215. doi:10.1037/0882-7974.12.2.203

Scheck, B., Neufeld, P., \& Dwyer, J. (2000). Actual innocence: Five days to execution, and other dispatches from the wrongly convicted. New York, NY: Doubleday.

Shipley, W. C. (1986). Shipley Institute of Living Scale. Los Angeles, CA: Western Psychological Services.

Tulving, E. (1985). Memory and consciousness. Canadian Psychology, 26, 1-12. doi:10.1037/h0080017

Valentine, T., Davis, J. P., Memon, A., \& Roberts, A. (2012). Live showups and their influence on a subsequent video line-up. Applied Cognitive Psychology, 26, 1-23. doi:10.1002/acp.1796

Wixted, J. T., \& Mickes, L. (2010). A continuous dual-process model of remember/know judgments. Psychological Review, 117, 10251054. doi: $10.1037 / \mathrm{a} 0020874$

Yonelinas, A. P., \& Parks, C. M. (2007). Receiver operating characteristics (ROCs) in recognition memory: A review. Psychological Bulletin, 133, 800-832. doi:10.1037/0033-2909.133.5.800 